\title{
ESPAÑA, DE RETAGUARDIA A OBJETIVO DEL TERRORISMO YIHADISTA (1990- 2012)
}

\author{
Mari Carmen Forriol Campos 1 \\ Universidad Católica de Valencia
}

http://dx.doi.org/10.5209/rev_NOMA.2013.v37.n1.42556

\begin{abstract}
Resumen.- Este trabajo pretende estudiar la evolución experimentada por el terrorismo yihadista en España durante el periódo comprendido entre los años 1990 y 2012, con la finalidad de profundizar en la comprensión de este fenómeno y en el nivel de amenaza que representa para nuestro país. Con este fin se examinaran los resultados de una muestra de 35 operaciones policiales realizadas por las Fuerzas de seguridad del Estado con la ayuda de la agencia de inteligencia de nuestro país durante el periódo comprendido entre 1990 y 2012, al objeto de conocer en que medida los grupos desarticulados están vinculados o no a organizaciones superiores y relacionados con la red del $11 \mathrm{M}$.
\end{abstract}

Palabras clave.- terrorismo yihadista, organizaciones superiores, vinculación, red del $11 \mathrm{M}$

\section{Spain from rearguard to objective of the yihadist terrorism (1990-2012)}

Abstract.- This work expect studies the evolution in Spain of the yihadist terrorism between 1990 and 2012, for the porpose to go deeply in the understanding of this phenomenon and in the level of threat for our country. With these purpose were examine the sucess of a sample of 35 police operations carried by the pólice forcé and the intelligence agency of our country during the period inter 1990 and 2012, in order to know to what extent the break up groups are linking or no to high organizations and related with $11 \mathrm{M}$ network.

Keywords.- jihadist terrorism, high organizations, link, the $11 \mathrm{M}$ network

\section{INTRODUCCIÓN}

En este trabajo no se pretende realizar una descripción detallada de cada una de las redes o grupos yihadistas establecidos en nuestro país durante el periódo comprendido entre los años 1990 y 2012 pues ya se trató en la Tesis doctoral titulada "El Terrorismo yihadista en Europa desde 1990 hasta 2012: desarrollo y aspectos organizativos"2sino hacer referencia a los relacionados con la red del $11 \mathrm{M}$.

\footnotetext{
${ }^{1}$ Licenciada en Arqueologia e Historia Antigua por la Universidad de Murcia, habiendo realizado en 1982 la Tesina titulada:"El material arqueológico de las villas romanas en la provincia de Murcia desde el siglo I antes de Cristo al siglo IV despues de Cristo." En 2006 comencé el doctorado realizando el trabajo del DEA sobre el "La financiación del terrorismo yihadista." En 2011 realicé un curso sobre Estudios Estrategicos y Seguridad Internacional. El 3 de mayo de 2013 defendí la Tesis doctoral titulada "El Terrorismo Yihadista en Europa desde 1990 hasta 2012: desarrollo y aspectos organizativos."

2 (2003) FORRIOL CAMPOS, Mari Carmen, "El Terrorismo Yihadista en Europa: Desarrollo y aspectos organizativos," Tesis doctoral dirigida por los doctores Pilar Pozo y Gines Marco.
} 
Para la realización de este estudio se ha recurrido principalmente a la documentación policial y judicial sobre investigaciones abiertas (Diligencias Previas, Sumarios, Sentencias), a trabajos monográficos, artículos publicados en revistas especializadas de carácter nacional e internacional, a noticias ofrecidas por la prensa impresa y digital nacional y extranjera, a los informes elaborados por Europol entre 2005 y 2012, a los anuarios sobre terrorismo yihadista publicados desde hace algunos años por el Ministerio del Interior español y a la información facilitada por la Tesis doctoral titulada "El Terrorismo Yihadista en Europa: Desarrollo y aspectos organizativos."

\section{El Terrorismo yihadista evoluciona en España de la retaguardia a la vanguardia}

Durante años las autoridades españolas y los especialistas de la Unidad Central de Información Exterior de la Policía y del Centro Nacional de Inteligencia quizás como consecuencia de su desconocimiento del terrorismo yihadista no entrevieron la amenaza que este fenómeno tendría para España. Un país que no sólo había acogido y otorgado una nueva nacionalidad a sus miembros, sino que les dejó transitar libremente y extender sus contactos en nuestro país y en el exterior. Fue a raíz de esta beligerancia como surgieron redes como la desarticulada en la operación Apreciate o la red de Abu Dahdah (la red de Al Qaeda en España), de las que formaron parte algunos de los integrantes de la red del $11 \mathrm{M}$, autora del mayor atentado ejecutado hasta el momento en Europa.

Se han dado varias versiones sobre el motivo por el que España fue en el atentado del $11 \mathrm{M}$ y posteriormente objetivo del terrorismo yihadista global. Una de ellas es que la ejecución de este atentado se llevó a cabo para recuperar el Al Ándalus a quien los ideólogos yihadistas consideran Dar al Islam (Tierra del Islam), un territorio que durante ocho siglos estuvo bajo poder musulmán y que en la actualidad está ocupado por infieles a los que se debe re islamizar a través de la yihad. Este en mi opinión es un aspecto que debe tenerse en cuenta aunque no es el principal, pues hay dos aspectos que alcanzan mayor relevancia y estos son: que España principalmente sea la puerta de entrada y salida de Europa y su presencia militar junto con EEUU y sus otros aliados en Tierras del Islam. Pues desde que el objetivo prioritario de Bin Laden fue realizar la yihad al enemigo lejano, su idea fue acabar con el poder de EEUU. Pero para acabar con su poder y su presencia en Tierras del Islam debía comenzar por atacar a sus aliados más vulnerables de la coalición. Una muestra de ello, es el documento publicado en una página web islamista radical en septiembre de 2003 en el que se describe la estrategia que Al Qaeda pensaba ejecutar para obligar a los aliados de EEUU a retirar sus tropas de Irak.

En este documento se da por sentado que resultaría infructuoso forzar la retirada de EEUU con la ejecución de nuevos atentados en suelo norteamericano, por lo que lo más eficaz sería atacar a sus aliados europeos en Irak, los cuales ante el temor de verse amenazados podrían verse obligados a retirar sus tropas de la zona. Lo que produciría un incremento de las cargas económicas para EEUU y dificultaría su ocupación de Afganistán, de Irak y de la Península Arábiga. En este documento se valoraba recurrir para 
coaccionarlos a la ejecución de atentados letales en España, Gran Bretaña y Polonia. De entre todos estos países se decidió comenzar por atacar a España que era el más vulnerable.

A continuación reproducimos fragmentos del documento en el que se confirma que los atentados se ejecutarían en España:

"Con el fin de obligar al gobierno español a que se retire de Irak, la resistencia debe provocar graves daños a sus fuerzas. Esto debe verse acompañado de una campaña de información donde se explique la verdad de lo que sucede en el interior de Irak. Es necesario aprovechar al máximo las próximas elecciones generales de España, que se celebraran en marzo del año que viene. Creemos que el gobierno español no podrá tolerar más de dos atentados, o a lo sumo tres, tras lo cual tendrá que retirarse como consecuencia de la presión popular. Si sus tropas permanecen en Irak después de tales atentados, es casi segura la victoria del Partido Socialista, que incluirá en su programa electoral la retirada de las fuerzas españolas. Por último, queremos recalcar que una retirada de las fuerzas españolas e italianas de Irak supondría una mayor presión para la presencia británica, presión que Tony Blair podría no soportar, lo cual provocaría de inmediato un efecto dominó. Aún así, persiste el problema fundamental, que consiste en provocar la caída de la primera.3

Tras los atentados del $11 \mathrm{M}$ y habiendo decidido España retirar las tropas de Irak, Bin Laden emitió otro comunicado en el que se comprometía a cesar los atentados contra aquellos países que decidiesen retirar sus fuerzas de Irak y Afganistán. Todos los aliados a excepción de España rechazaron la oferta.

Este comunicado decía:

"Anuncio una tregua con los países europeos que no ataquen a los países musulmanes (...) La puerta hacia la tregua estará abierta durante tres meses (...) La tregua comenzará cuando el último soldado abandone nuestros países (Irak y Afganistán). (...) Se dice que matamos por matar, pero la realidad demuestra que es falso (...) La matanza de rusos sólo comenzó después de su ataque contra Afganistán en los años ochenta y contra Chechenia, la de los europeos después de la invasión de Irak y Afganistán (,) y la de los norteamericanos en Nueva York después del apoyo de los judíos en Palestina y la invasión de la península arábiga. Dejen de derramar sangre de los nuestros y nosotros dejaremos de derramar la de los suyos (15 de abril de 2004)."4

¿Se puede considerar que España se convirtió en objetivo prioritario del terrorismo yihadista global por su participación en la guerra de Irak? Parece evidente a la vista de estos documentos que esta afirmación es la más adecuada, aunque ello no significa que no haya que tener en cuenta la versión que defiende que nuestro país fue atacado porque desde hace años forma parte "del imaginario obsesivo de la ideología islamista." 5

\footnotetext{
3 "Jihadi Iraq, hopes and dangers," documento dedicado a Yusuf al-Ayiri. Publicado originariamente en la página web "Global Islamic Media" y posteriormente en la página web del Forswarets Forskningsinstitutt, Instituto noruego de investigación para la defensa.

${ }^{4}$ (2004) Fragmento de una cinta de Bin Laden, USA Today, 15 de abril de 2004.

${ }^{5}$ (2005) DE ARÍSTEGUI, Gustavo, La Yihad en España. La obsesión por reconquistar AlÁndalus, Ed. La Esfera de los libros, $4^{\mathrm{a}}$ edición, p. 10.
} 
Una vez analizados los motivos por los que el terrorismo yihadista global eligió nuestro país para ejecutar los atentados del $11 \mathrm{M}$, estudiaremos por qué España ha sido desde 1990 hasta 2012 un lugar de gran atracción para las organizaciones yihadistas. Está claro que a ello, han contribuido una serie de factores como es su proximidad geográfica con los países del Magreb, su facilidad de comunicación con Francia e Italia en donde operan grupos yihadistas magrebíes, la elevada densidad de tráfico de personas y mercancías en el Estrecho, la conexión marítima directa entre Argelia y España a través de los puertos de Alicante, Valencia y Barcelona. Factores todos ellos que han ayudado a los yihadistas a infiltrarse en nuestro país sin levantar sospechas y a establecer una base de reclutamiento, de financiación y de apoyo logístico para los grupos yihadistas del interior y a los establecidos en Europa, Argelia y Asia Central.

Con el fin de estudiar la evolución y el desarrollo del terrorismo yihadista en España se realizará un análisis de los grupos yihadistas establecidos en nuestro país desde 1990 hasta 2012. Para facilitar su explicación realizaremos un estudio en primer lugar del material recogido sobre la muestra de nueve operaciones policiales llevadas a cabo durante el periodo comprendido entre 1990 y 2003, haciendo especial referencia a los grupos relacionados con la red del $11 \mathrm{M}$. En segundo lugar, se realizará un análisis de las treinta y cinco detenciones realizadas durante 2004 y 2012, sin incluir a la red del $11 \mathrm{M}$ a la que se dedicará un apartado especial.

Con respecto a las detenciones llevadas a cabo antes del $11 \mathrm{M}$ se observa que la mayoría de los yihadistas establecidos en nuestro país entre la última década de 1990 y 2003 procedían de Siria lugar de origen de gran parte de los componentes de la red de Abu Dahdah. Asi mismo, un gran número procedía de Argelia y de Marruecos dos lugares cercanos a la Península

La mayoría de estos grupos se establecieron en las CCAA de Cataluña, Madrid, Andalucía, Valencia y Murcia, lugares de fácil acceso al empleo, en los que se presentaban las mejores ofertas de vivienda y en los que se concentraba población argelina, siria y marroquí.

Estos grupos y redes durante este periódo, se dedicaron a desempeñar tareas de apoyo logístico, financiero y de reclutamiento, pero nunca atentaron en nuestro país. Uno de los mejores ejemplos es la celula de Abu Dahdah. Un grupo que desempeñó una floreciente actividad proselitista entre los jóvenes de primera generación, a los que una vez reclutadados los enviaba a que fuesen entrenados a los campamentos de Al Qaeda en Bosnia y en Afganistan o como voluntarios a los frentes de Bosnia, Chechenia e Indonesia. 6 En sus orígenes la labor de reclutamiento se llevó a cabo generalmente en las mezquitas. Por ejemplo, Abu Dahdah era uno de los que acudía a estos focos de reclutamiento para establecer contacto y distribuir propaganda entre los asistentes. 7 Cuando los imanes moderados se dieron cuenta de la labor que realizaban se lo prohibieron, por lo que decidieron esperar a los fieles en el exterior y cuando estos salían, se fijaban en los que mostraban mostraban interés en la propaganda distribuida, con el fin de establecer un primer contacto e invitarles a disfrutar de un día de excursión con sus familias y reunirse para dialogar

\footnotetext{
${ }^{6}$ Sumario 35/01, $\mathrm{JCl} \mathrm{n}^{0}$ 5, Sala de lo Penal, Sección $3^{\mathrm{a}}$ de la AN, p. 25, 2001.

${ }^{7}$ Sentencia 36/05, Sala de lo Penal, Sección 3a de la AN, p.25-30, 26 de septiembre de 2005.
} 
sobre la situación del Islam en el mundo.8 Cuando el reclutador observaba en alguno de los asistentes una cierta inquietud por la causa, le invitaba a reunirse con un reducido grupo de jóvenes en un domicilio particular, en la que se les animaba a radicalizarse. En otras ocasiones el reclutamiento se iniciaba en movimientos islamistas musulmanes como Hizb ut Tahrir o Yama'a al Tablig en donde por ejemplo Abu Dahdah conoció a Amer Azizi quien reclutó posteriormente a Maymouni

Otra de las actividades a las que se dedicó esta red y la mayoría de las desarticuladas durante este primer periódo, es a la financiación del propio grupo y de otros grupos del interior y del exterior del país. Financiación que conseguían principalmente a través de los donativos que entregaban sus miembros y de actividades delictivas como el robo. Con el dinero conseguido no sólo financiaban los viajes de sus miembros y de los voluntarios que marchaban a lugares de conflicto sino que sufragaban el alquiler de los pisos o casas en las que residían, el material que necesitaban para elaborar la documentación falsa, para facilitar la asistencia médica a los mujahidines y a los componentes de la red terrorista internacional que la necesitasen. 9 Una gran proporción de los fondos recaudados en el caso de la red de Abu Dahdah eran entregados por su líder a Abu Qatada para que los distribuyese entre los grupos más necesitados10 y para cubrir las necesidades de los mujahidines. Otra de las actividades importantes de esta red es la falsificación de documentos (pasaportes, visados de residencia, tarjetas de trabajo y tarjetas de crédito que robaba una pequeña red de delincuentes externa al grupo). Una documentación que se distribuían a los integrantes de los grupos del interior y del exterior con el fin de que pudiesen transitar libremente por Europa, viajar a los campos de entrenamiento y a los lugares de conflicto. Una actividad que continuaron ejerciendo la mayoría de los grupos desarticulados entre los años 2004 y 2012.

Los mayoría de los grupos desarticulados durante este periódo se caracterizaron por ser grupos vinculados al GIA, al GSPC y a Al Qaeda, a excepción de la célula dirigida por Samir Mahjoub que lo estuvo a Ansar al Islam.11

De entre estos grupos los componentes del desarticulado en la operación policial Lago y los de la red de Abu Dahdah mantuvieron no sólo relación con líderes y miembros destacados de grupos yihadistas establecidos en el interior sino también con los del exterior. Así por ejemplo, en la red de Abu Dahdah su líder y Taysir Aloun, el líder del subgrupo de esta red en Granada, sostuvieron contacto personal con miembros de Al Qaeda repartidos por el mundo. El segundo mantuvo contacto habitual con Abu Qutada y con Mohamed Bahaiah y Setmarian a los que les unía una gran amistad. Mientras que el primero como

\footnotetext{
${ }^{8}$ (2006) JORDÁN, J, MAÑAS, F.M, TRUJILLO, H, "Pérfil sociocomportamental y estructura organizativa de la militancia yihadista en España. Analisis de las redes de Abu Dahdah y del 11 M," Inteligencia Seguridad, Revista de Analisis y Perspectiva, $n^{\circ}$ 1, p. 79-111

${ }^{9}$ Sumario 35/01,...op. cit. en nota 1, p. 26, 2001.

${ }^{10}$ En uno de los registros realizados al domicilio de Abu Qutada el 13 de febrero de 2001, se encontró la suma de más de 5100000 pesetas en monedas y una cantidad superior a esta se encontró guardada en sobres y bolsas que llevaban escrito el destino del dinero (Sumario $35 / 01 \ldots$ op. cit., en nota 2, p. 192).

${ }^{11}(2004)$ YOLDI, José, "Garzón encarcela a los detenidos por integración en Ansar al Islam," El País, Madrid, 20 de mayo de 2004.
} 
líder de la red mantuvo estrecha relación con figuras relevantes dentro de Al Qaeda en Europa como Abu Qutada, coordinador de Al Qaeda en Europa y responsable de los coordinadores locales y regionales ubicados en Europa además de ser coordinador del GSPC, del GICM y del Frente Islámico Túnecino. Tambien estableció estrecha amistad Mohamed Bahaiah que fue el responsable en Al Qaeda de establecer y mantener los contactos a nivel internacional. A partir de 1995 sostuvo una estrecha amistad con Mustafa Setmarian, una amistad que se mantuvo durante su estancia en Londres y posteriormente en Afganistan. Estuvo también en constante contacto con miembros destacados de Al Qaeda como Salaheddine Benyaich, Mamoun Darkanzali y con Zaival Abideen Muhammad Asan el responsable de Al Qaeda a escala mundial en cuanto a la organización, logística y coordinación de los mujahidines. Existe un más que probable indicio de que se relacionase con los integrantes de la célula de Hamburgo, en concreto con Mohamed Atta, Ramzi Bin Al Shibh, Said Bahaji y con un sujeto identificado como Shakur, quien desde el Reino Unido le mantuvo informado de los preparativos del $11 \mathrm{~S}$. Además de estas relaciones y contactos a nivel internacional, cabe destacar su relación y la del grupo desarticulado en la operación Apreciate con la red del $11 \mathrm{M}$. Relación que en el caso de este segundo grupo se realiza a través de Allekema Lamari y Sohi Khouni, autores materiales de los atentados y que en el caso de la red de Abu Dahdah se realiza a través de Driss Chebli y Amer Azizi, reclutador de Mustafa Maymouni y del Tunecino, líderes del grupo Villaverde, núcleo originario de la red del $11 \mathrm{M}$.

Una vez analizado el desarrollo del terrorismo yihadista en España en el periodo comprendido entre el año 1990 y 2003, se pasará a estudiar el desarrollo de los grupos de inspiración yihadista establecidos en nuestro país en el periodo comprendido entre 2004 y 2012 con exclusión de la red del 11 M a la que como se afirmó anteriormente se dedicará un apartado especial.

Una vez analizado el desarrollo del terrorismo yihadista durante los años comprendidos entre 1990 y 2003, se realizará un estudio de su evolución de los grupos desarticulados entre 2004 y 2012. Para ello, se ha realizado una selección de las operaciones policiales efectuadas durante este periodo, partiendo del análisis descriptivo realizado en la Tesis doctoral titulada "El Terrorismo yihadista en Europa desde 1990 hasta 2012: Desarrollo y aspectos organizativos."

De forma especial se analizará la procedencia geográfica y las actividades realizadas por los componentes de los grupos desarticulados durante estos limites temporales, asi como su vinculación con las organizaciones superiores y con la red del $11 \mathrm{M}$.

De las treinta operaciones policiales llevadas a cabo durante estos años se observa que la mayoría de los componentes de estos grupos yihadistas son de procedencia magrebí, lo que coincide con una de las nacionalidades de los integrantes de los grupos desarticulados durante el periodo anterior. A esta hay que sumar los conversos españoles y los de nacionalidad paquistaní representada en los grupos paquistaníes desarticulados en Barcelona en los años 2004 y 2008.

A diferencia de los grupos desarticulados en la epóca anterior, la mayoría de los grupos desmantelados durante estos años se establecieron en Cataluña, al 
parecer porque es el lugar donde se concentra mayor población magrebí o quizás porque las autoridades locales no toman las medidas necesarias para evitar que los yihadistas se inflitren en este sector poblacional y realicen una extensa labor proselitista con el objeto de conseguir nuevos adeptos. Tambien puede deberse como afirma el profesor Jordán12 a la cercanía de Cataluña a la frontera, lo que posibilitaría una fácil y rápida comunicación con los grupos yihadistas establecidos en Francia e Italia.

Como en el periódo anterior la mayoría de los integrantes de los grupos desarticulados en este periódo realizaban actividades logísticas y financieras como las que se llevaron a cabo en apoyo de los autores huidos de los atentados del $11 \mathrm{M}$ y las actividades de falsificación de documentos y de reclutamiento. Una actividad esta que a diferencia de la de la etapa anterior se realiza principalmente a través de internet y en/desde las prisiones como sucedió en el caso del grupo de Topas desarticulado durante las operaciones Nova I y Nova II. Este reclutamiento también se realiza en sociedades paralelas como el barrio Principe Alfonso en Ceuta, en los cibercafés, en los halalai, en los centros educativos, en locales comerciales, en locutorios, en centros asistenciales, centros de acogida, gimnasios, en carnicerías y en pollerías como la de Merabet Farsi. A estas actividades hay que sumar la de reclutar jovenes voluntarios que serían enviados a engrosar las filas de organizaciones Al Qaeda en Irak como la realizada por el grupo de Vilanova y como Geltrú, lo que en ocasiones se simultaneaba con el envío previo a Argelia de voluntarios que posteriormente serían enviados a Irak.

El no haber hecho referencia en el periódo anterior a la preparación de los atentados no significa que no se planificasen, pues si se toman como ejemplo los atentados del $11 \mathrm{M}$ y los de Casablanca se comprobará que estos no se planearon con tan sólo unos meses de antelación sino en el año 2002 durante una reunión mantenida en Estambul. Esta decisión fue tomada por los líderes de AI Qaeda, el GICL y el GICM, que decidieron que había llegado el momento en que los musulmanes residentes en Occidente debían comenzar a atacar los países en los que residían. Fue en ese momento cuando Maymouni (líder de la célula de Kenitra y del grupo de Villaverde) recibió la orden de realizar los atentados de Casablanca que se ejecutaron en mayo de 2003. Actitud de la que tomaron ejemplo grupos como el de Topas que planificó el atentado contra la Audiencia Nacional, la célula que programó el atentado contra el ferry que enlaza Ceuta y Algeciras o los ataques que intentaron llevar a cabo las células paquistaníes en Barcelona en los años 2004 y 2008.

Al objeto de determinar si existe o no vinculación entre los grupos establecidos en España entre los años 2004 y 2012 con las organizaciones superiores se ha efectuado un análisis de los contactos habituales y de la relación establecida entre los operativos de las organizaciones superiores y los líderes o miembros destacados de estos grupos y la coordinación directa o a través de los coordinadores, de estos grupos con otros relacionados con la misma organización. Lo que no ha resultado fácil especialmente cuando los líderes o miembros destacados de un grupo están vinculados a más de una organización, como sucede en el caso de Mohamed Beldhadj que además de

\footnotetext{
${ }^{12}$ (2009) JORDÁN ENAMORADO, Javier, "El terrorismo yihadista en España: evolución despues del $11 \mathrm{M}$," Documento de Trabajo, $\mathrm{n}^{\circ} 7$.
} 
ser uno de los integrantes de la célula belga del GICM era portavoz de Al Qaeda en Europa o el de Larbi Ben Sellam que además de pertenecer al grupo Villaverde pertenecía a la célula mixta integrada en la red Tigris.

De entre todas las detenciones llevadas a cabo entre 2004 y 2012 en nuestro país, se puede afirmar que tan sólo una responde a la categoría de lobo solitario y es el caso del franco marroquí Larfi, que fue detenido por Los Mossos d'Esquadra mientras viajaba en un coche cargado de bombonas de butano, con material pirotécnico y una espada ninja ajustada horizontalmente desde el asiento trasero a la espada del conductor con el fin de suicidarse. La datación de este único caso demuestra que esta es una figura anómala, pues el terrorismo yihadista es una actividad colectiva. De hecho, la mayor parte de los grupos establecidos en España tras el $11 \mathrm{M}$ estuvieron coordinados con grupos del interior y de fuera de nuestro país y vinculados a organizaciones superiores como el GSPC, Al Qaeda, el GICM, a AQMI, Al Qaeda en Irak, dos lo estuvieron a Ansar al Islam, las células paquistaníes desarticuladas en Barcelona en 2004 y 2008 lo estuvieron a las organizaciones Jaish e Mohammed y Tehrik e Taliban y la desarticulada en la operación Duna lo estuvo a la organización Salafia Yihadia.

De entre las treinta detenciones realizadas entre 2004 y 2012 tan sólo cuatro de los grupos preparaban atentados, mientras que el resto se dedicaba a llevar a cabo actividades logísticas, financieras, a la difusión de propaganda y al reclutamiento de nuevos miembros con el firme propósito de enviarlos como voluntarios a Irak.

Si se hace referencia a los grupos desarticulados tras el $11 \mathrm{M}$ que tuvieron relación o prstaron ayuda de alguna forma a esta red, hay que afirmar que los líderes o miembros destacados de siete de los grupos desarticulados entre 2004 y 2012 estuvieron relacionados o apoyaron a sus miembros. De entre estos cabe destacar a Mohammad Afzaal, el líder de la célula paquistaní desarticulada en Barcelona en 2004 por su estrecha relación con Mohamed El Egipcio.13 A Kalid Zeimi Pardo, detenido en el marco de la Operación Nova, que mantuvo una estrecha relación con Amer Azizi, con el "Tunecino," con Said Berraj, Fouad El Morabit, con Basel Ghalyoun y con Mohamed el Ouzzani. Del mismo modo los trece detenidos durante la operación Saeta el 1 de abril de 2005 mantuvieron relacion con algunos integrantes de la red del $11 \mathrm{M}$ y con algunos de los asistentes a las reuniones celebradas por la célula Virgen del Coro. Asi mismo, algunos miembros del grupo "Martires de Marruecos" guardaron estrecha relación con algunos autores de los atentados de Casablanca y de los del $11 \mathrm{M}$.

Si atendemos al apoyo prestado a esta red por los líderes o miembros destacados de los grupos desarticulados tras el $11 \mathrm{M}$, se constata que Abdallah Brouka, líder de la célula desarticulada en la operación Frontón, mantuvo escondido en su casa a Hamal Ahmidan a quien le unía una estrecha amistad, mientras se recuperaba de una herida originada en una reyerta. Asi mismo, el grupo desartuculado en la operación Green en diciembre de 2005 y la célula de Santa Coloma de Gramanet ayudaron a los huidos de la célula del $11 \mathrm{M}$ (Mohamed Afallah, Mohamed Beldhadj, Daoud Ouhnanes, Said Berraj, Othman

\footnotetext{
${ }^{13}$ Mohamed El Egipcio fue acusado en Italia de ser uno de los responsables de los atentados del $11 \mathrm{M}$
} 
el Mouhib y Abdelilah Hriz) alojandolos, procurándoles ayuda económica y preparando algunos planes de huida que facilitasen su entrada en Irak. Asi mismo, en el Sumario 49/09 queda explicitado que el líder de esta célula, Samir Tahtah, que había entablado una estrecha amistad con los autores de los atentados del $11 \mathrm{M}$ huidos durante su estancia en el inmueble de Al Kaal, les prestó apoyo economico en su marcha hacia Irak. Esta ayuda hace referencia al envío de dinero que realizó a Turquía por medio de transferencias desde algunas de las operadoras de la Western Union en Santa Coloma de Gramanet,14 con el propósito de que emplease en adquirir documentación falsificada, billetes de viaje, alimentación y cobijo, a los huidos mientras esperaban ser enviados a Irak. Además de estas emisiones de dinero, realizó otras por medio de Hammad Lahsini a Mohamed Beldhadj durante su estancia en Belgica. Filali Oulali como coordinador del grupo de Santa Coloma jugó también un papel relevante en la ayuda a los autores huidos de los atentados del $11 \mathrm{M}$ al facilitarles documentación falsificada con el fin de que pudiesen cruzar fronteras sin ser detectados. Del mismo modo cinco de los integrantes de la red Tigris colaboraron con Mohamed Beldhadj, Mohamed Afallah y Doud Ouhnan. Larbi Ben Sellam se encargaó de coordinar la fuga de Mohamed Afallah y de Mohamed Beldhadj. Tambien los detenidos durante la operación Suez el 6 de noviembre de 2006 se encargaron de elaborar documentos falsos para Jamal Ahmidan y Abdennabi Kounjaa.

Los resultados del análisis realizado sobre estos grupos nos permite establecer que un número notable de estos mantuvieron relacion con la red del $11 \mathrm{M}$ y con algunos de los componentes del yihadismo internacional, lo que conduce a la conclusión de que la red del $11 \mathrm{M}$ es una red vinculada y no independiente como se ha defendido durante mucho tiempo.

De entre estas treinta y cinco detenciones efectuadas por las fuerzas de seguridad del estado entre 1990 y 2012 concluimos que la mayoría de los grupos yihadistas establecidos en nuestro país están vinculados a organizaciones superiores, lo que no impide que existan células independientes como la de Topas.

La mayoría de los grupos yihadistas establecidos en nuestro país durante la última década del siglo XX estuvieron relacionados principalmente con el GIA y el GSPC, mientras que los grupos yihadistas establecidos en nuestro suelo durante los primeros años del siglo XXI estuvieron vinculados a Al Qaeda, al GICM, a AQMI, a Lashkar e Taiba y en el caso de las células paquistaníes que pensaban atentar en Barcelona en 2004 y 2008 estas estuvieron vinculadas a Jaish e Mohammed y a la organización Tehrik e Taliban.

\section{España de retaguardia a objetivo de la red del $11 \mathrm{M}$}

A lo largo de estos trece años se han publicado libros en los que se ha escrito sobre ciertas teorías de conspiración. Libros que han sido públicados con el único fin de atraer la atención de la opinión pública española y con el firme objetivo de ocultar lo que realmente sucedió.

\footnotetext{
${ }^{14}$ SUMARIO 49/09, JCI no 5, Sección Segunda de la Sala de lo Penal de la AN, p. 76-78.
} 
Lo que ha generado que a estas alturas seamos muy pocos los que nos preocupemos por averiguar si la red del $11 \mathrm{M}$ es una célula independiente 0 está vinculada a algunas de las organizaciones superiores del terrorismo yihadista global y a descubrirlo es a lo que se dedica este apartado.

Al objeto de conocer a fondo si esta red es un grupo vinculado o no, se comenzará por realizar un análisis de su origen, desarrollo y de los contactos y establecidos directamente o a través de coordinadores entre los líderes y destacados miembros de la red con grupos del interior y del exterior y con las organizaciones superiores.

Establecer el momento preciso en que surgió esta red resulta difícil pero de lo que no cabe duda es que a raiz de la operación Dátil llevada a cabo contra la célula de Abu Dahdah en noviembre de 2001 comenzó a organizarse la red.

Amer Azizi, lugar teniente de Abu Dahdah en la célula de Al Qaeda en España fue uno de los no detenidos pues en aquel momento se encontraba en un campo de entrenamiento de Afganistan del GICL. Fue allí donde se adscribió al GICL y estableció una relación muy estrecha con relevantes miembros de esta organización y del GICM. Al enterarse de las detenciones realizadas durante la operación Dátil regresó a España, donde con la ayuda de Mustafa Maymouni que había sido reclutado por él, se dedicó con todos sus esfuerzos a reunir a los miembros de la red de Abu Dahdah que habían quedado en libertad en nuestro país y a captar nuevos jóvenes que se unieron a lo que fue núcleo originario (la célula de Villaverde) de la red del $11 \mathrm{M}$. Una vez organizado este grupo, Amer Azizi se vió obligado a huir de España y el relevo en la dirección de esta celula lo tomaron Maymouni, Driss Chebli y Sarhane Ben Abdelmajid Fakhet, los cuales con el fin de potenciar el grupo dedicaron todas sus esfuerzos en realizar una intensa labor proselitista.15 Tras la detención de Maymouni y Driss Chebli por su participación en la ejecución de los atentados de Casablanca se quedó como líder del grupo Sarhane que tambien había sido reclutado por Amer Azizi.

Por otra parte, la detención de un elevado número de componentes de la red de Abu Dahdah, incluido su líder y la de su lugarteniente Said Chedadi, movilizó a los comerciantes del barrio de Lavapiés a reagruparse y organizar bajo la dirección del marroquí Zougam el denominado grupo de Lavapiés, que mantuvo estrecha relación con figuras del terrorismo yihadista internacional como David Coutailler, Abu Hamza al Masri, Mullah Krekar que por entonces era líder espiritual de Ansar al Islam.

El tercer grupo (grupo de la Virgen del Coro) realizaba las reuniones de adoctrinamiento en un local ubicado en la calle Virgen del Coro, de ahí el nombre del grupo. Un local arrendado por Mouhammad Moutaz quien junto con su hermano Moutaz se dedicó al reclutamiento de jóvenes musulmanes a través de mezquitas como la de Abu Bakr y la M 30. Fue a través de algunos de estos jóvenes como conocieron a marroquíes que como Jamal Ahmidan procedían del mundo de la delincuencia y a otros como El Mourabib Anghar y Basel Ghalyoun a los que ofrecieron ese local para residir.16

\footnotetext{
${ }^{15}$ SUMARIO 20/04, JCl n6, Sección Segunda de la Sala de lo Penal de la AN, p.1344.

${ }^{16}$ SENTENCIA 36/05, op. cit...en nota 1, p. 25-30, 26 de septiembre de 2005.
} 
Rabei Osman a quien se considera uno de los autores intelectuales del $11 \mathrm{M}$, fue el líder de este grupo y a su vez el coordinador de este grupo y el de Villaverde y como tal mantuvo una estrecha relación con Sarhane y Zougam. Pero su relación no sólo se centró en estos sujetos sino que se extendió a los integrantes de una red marroquí que fue desarticulada mientras se preparaba para ejecutar atentados en Belgica y en Francia, a Mohammed Rahed a quien reclutó y a Mourad Chabarou que fue el que refugió en su casa de Bruselas a Mohamed Afallah y a Mohamed Beldhadj.

A estos tres grupos se les unió el cluster dirigido por Hamal Ahmidan, compuesto por delincuentes. Este no es el primer caso en el que una red yihadista se relaciona con la delincuencia en España, pues anteriormente la red de Abu Dahdah también había mantenido relación con un grupo de delincuentes aunque en este caso estos no formaban parte de la red de Al Qaeda en España.

El cluster de Hamal Ahmidan era un grupo conformado por criminales profesionales que se convirtieron en yihadistas tras incorporarse a la red del 11 $M$ en 2003. Estos por su relación con el tráfico de droga se responsabilizaron de conseguir los explosivos del grupo de Asturias para ejecutar los atentados y de sufragar el alquiler de pisos francos. Una actividad propia de una célula independiente y que quizás es lo que ha llevado durante años por falta de información policial y judicial a algunos expertos a considerarla como célula independiente. Pero tener en cuenta sólo este aspecto no es suficiente para poder catalogar a una red como no vinculada a una organización superior, pues tambien las células vinculadas a organizaciones superiores gozan de un elevado grado de autonomía y se financian a través de delitos comúnes como es el caso de la red de Abu Dahdah, la red de Al Qaeda en España.

Este grupo estableció contacto con el grupo de "Avilés" a principios de 2003 por mediación de Zouhier que fue el intermediario entre Hamal Ahmidan y Suarez Trashorras que fue el que consiguió los explosivos y los detonadores, los cuales eran entregados a Hamal Ahmidan.

La célula autora de los atentados del 11 M quedó configurada en 2003 con Hamal Ahmidan, Mohamed Oulad Akcha, Rachid Oulad Akcha, Abdennabi Kounjaa, Ashrif Rifaat Anour, Allekema Lamari,17 Jamal Zougam, Allekema Lamari, Doud Ouhnane, Abdelmajid Bouchar y Mohamed Afallah. Casi todos formaban parte del yihadismo global antes de conformarse la red del $11 \mathrm{M}$.

Una vez constituido el grupo se reunieron en dos ocasiones con Rabei Osman para recibir las últimas instrucciones sobre el atentado. Este fue el que desde Italia, el 4 de febrero activó su buzon de correo Kishkmommed@yahoo.com para comunicar a través de una fecha de nacimiento, el día y el mes en que deberían llevarse a cabo los atentados.

Atentados en los que no se empleó el método suicida. Una táctica que en cambio si emplearon algunos de los autores materiales el 3 de abril en Leganés al ser descubiertos por la Unidad Central de Información Exterior.

Tras haber expuesto de una forma breve el origen, desarrollo y estructura de la red del $11 \mathrm{M}$, se procederá a analizar el apoyo y la relación que esta red obtuvo

${ }^{17}$ SENTENCIA 65/07...op.cit. en nota 1, p.576. 
de organizaciones superiores como Al Qaeda, el GICM y el GICL y de grupos del interior y del exterior a relacionados con estas, con el objeto de mostrar su vinculación a una organización superior.

La relación de esta red con Al Qaeda se realiza a través de la red de Abu Dahdah a la que cabe considerar el origen remoto de la red del $11 \mathrm{M}$ pues de ella formó parte Amer Azizi, lugarteniente de Abu Dahdah, reclutador de Maymouni y de Sarhane y organizador junto con el primero del núcleo originario de la red del $11 \mathrm{M}$. Un grupo que en un principio estuvo liderado por Maymouni, Driss Chebli y Sarhane, pero tras la detención de los dos primeros por su participación en los atentados de Casablanca en mayo de 2003, quedó a cargo del grupo Sarhane.

Amer Azizi tras permanecer entrenando en uno de los campos de entrenamiento adscritos al GICL donde conoció y contactó con elevados cargos del GICM como Karim El Majjati y Mohamed y Kamal Chatbi y con miembros del GICL como Malek el Andalusi, acabó por unirse al GICL. Fue como consecuencia de esta unión al GICL y de su relación con el GICM, como alcanzó un lugar destacado en el mando de operaciones externas de Al Qaeda.

Otro de los nexos de unión de la red del $11 \mathrm{M}$ con Al Qaeda se realiza a través con la marcha de Moutaz Almallah al Reino Unido donde trabaja con Abu Qutada, quien no sólo era el líder espiritual del GIA, del GSPC, del Grupo Combatiente Tunecino y de Al Qaeda en Europa, sino que se encargaba de la financiación de los atentados de Al Qaeda en Europa.

La vinculación de la red del $11 \mathrm{M}$ con el GICM en Europa surge del apoyo prestado por algunos líderes y destacados miembros de esta organización superior a la red en lo que respecta a la preparación, planificación y huida de algunos de los autores materiales.

Hassan el Haski (líder del GICM en Europa) con Rabei Osman, Joussef Beldhadj y Sarhane18 uno de los autores intelectuales de los atentados del 11 M.19 Sarhane fue el que en el verano de 2003 una vez planificados los atentados, presentó a Amer Azizi el proyecto de los atentados del $11 \mathrm{M}$ para su aprobación por la cúpula de Al Qaeda. Una vez aprobado, Sarhane pidió a Azizi que le proporcionase militantes del GICM para constituir la célula autora de los atentados, a lo que Azizi se negó. Porque la mayoría de los miembros pertenecientes a esta organización se encontraban encarcelados 0 responsabilizados de otras actividades.

Pero la conexión con el GICM no se realiza sólo a través de Amer Azizi y de Hassan el Haski, sino principalmente a través de Youssef Beldhadj y de Chabarou, pertenecientes a la célula belga del GICM.20

Youssef Beldhadj además de ser un destacado miembro del GICM belga era el portavoz de Al Qaeda en Europa, el que impartió las últimas instrucciones sobre los atentados y el que con su hermano Mimoun preparó tras los atentados la huida a Irak de Mohamed Beldhadj y de Mohamed Afallah a quien había reclutado durante su estancia en Leganés. Mientras que Chabarou se

\footnotetext{
${ }^{18}$ SUMARIO 20/04...op. cit. en nota 1, p. 197

${ }^{19}$ SENTENCIA 65/07...op. cit en nota 1, p. 565.

${ }^{20}$ SUMARIO 20/04...op. cit en nota 2, p. 1271.
} 
responsabilizó de cobijar a los anteriores en su domicilio en Bruselas durante su estancia la ciudad.

Por su parte la celula francesa del GICM apoyó a la red del 11 M escondiendo a algunos autores huidos de los atentados y la financió con la entrega de cantidades dinerarias a Rabei Osman.

Tambien la red española del GICM ayudó a los autores huidos de los atentados del $11 \mathrm{M}$ proporcionándoles el medio de transporte para llegar a Bélgica a donde se les transfirió frecuentes sumas de dinero y consiguiéndoles documentación falsa a través de Filali Ouali.

Es a traves del apoyo recibido por estas organizaciones y de la relación de suslíderes y destacados miembros con los de la rd del $11 \mathrm{M}$ y de estos con algunos de los grupos desarticulados tras el $11 \mathrm{M}$ en España a los que hemos hecho referencia con anterioridad, como hemos llegado a la conclusión de que la red del $11 \mathrm{M}$ no es un grupo independiente sino vinculado ideológicamente, en su liderazgo y estrategia a organizaciones superiores como Al Qaeda, el GICM y el GICL. 


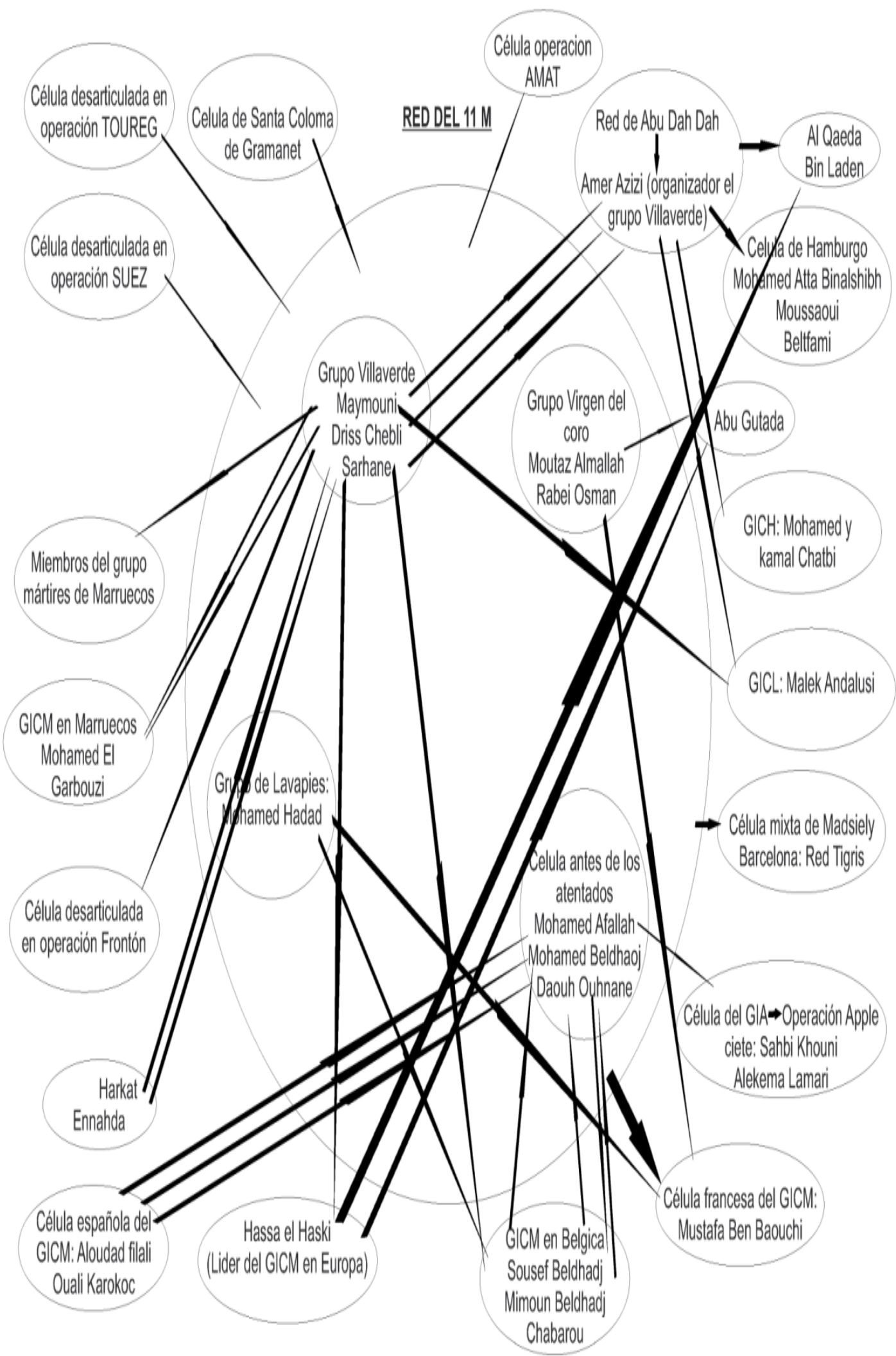

Grafo que muestra la vinculación de la red del $11 \mathrm{M}$ con algunas organizaciones superiores y con grupos establecidos en el exterior e interior de España. (Elaboración propia) 


\section{CONCLUSIONES}

Los grupos yihadistas adscritos al Terrorismo yihadista en España en el periodo comprendido entre 1990 y 2004, se dedicaron especialmente a tareas de apoyo logístico y financiero a grupos del interior y del exterior, a la difusión de propaganda y al reclutamiento de nuevos miembros que durante estos años se realizaba a través de las mezquitas y de movimientos islamistas que como El Tabligh que aunque no promueven la violencia contribuyen indirectamente a la radicalización.

Desde 2004 estos grupos se dedican a las tareas emprendidas en la etapa anterior aunque con alguna variación como sucede en el caso del reclutamiento en el que el lugar de las mezquitas lo ocupan las prisiones e internet. Otra de las diferencias con respecto a la época anterior es que los voluntarios que anteriormente eran enviados a entrenar o a combatir a Bosnia, Chechenia y a Afganistan, a partir de 2004 marchan a Irak a engrosar las filas de Al Qaeda en Irak. Es en esta etapa cuando los grupos animados por la ejecución de los atentados del $11 \mathrm{M}$, comienzan a intentar ejecutar atentados como el que pretendía realizar el grupo de Topas contra la Audiencia Nacional.

En su mayoría los grupos establecidos en España desde 1900 hasta 2004 estuvieron vinculados durante la primera mitad de la última década del siglo $X X$ al GIA, mientras que en la segunda mitad lo estuvieron al GSPC, a Al Qaeda y al GICM.

Desde 2004 la mayoría de los grupos se encontraban vinculados a AQMI, al GICM y a Al Qaeda y en el caso de los grupos paquistaníes desarticulados durante 2004 y 2008 lo estuvieron a organizaciones como Tehrik e Taliban y a Lashkar e Taiba.

Como hemos afirmado anteriormente, la red del $11 \mathrm{M}$ no es una red independiente sino vinculada en su liderazgo, en su ideología y estratégicamente a organizaciones sueriores como Al Qaeda, el GICM y el GICL y con grupos establecidos en el interior y exterior de nuestro país.

Por último cabe destacar que España no es un país en el que los lobos solitarios hayan tenido relevancia pues solo tenemos documentado un caso, lo que indica que esta es una figura anómala en nuestro país. 